\title{
EFICACIA DE COAGULANTES EN EL TRATAMIENTO PRIMARIO DE EFLUENTES DE PROCESADORA DE FRUTAS
}

\author{
Effectiveness of coagulants in primary treatment of effluents from processing fruits \\ ${ }^{1}$ Mario Alcarraz C, ${ }^{2}$ Gerardo Gamarra B, ${ }^{2}$ Américo Castro L, ${ }^{2}$ Juan Godoy A \\ ${ }^{1}$ Laboratorio de Bioprocesos Industriales - Facultad de Ciencia Biológicas, ${ }^{2}$ Facultad de Farmacia y Bioquímica \\ Universidad Nacional Mayor de San Marcos
}

\section{RESUMEN}

Los efluentes industriales sin tratamiento previo ni control de las autoridades contaminan el medio ambiente. Resolver el problema planteado no pasa solamente por el control, supervisión y sanción a las empresas generadoras de efluentes, requieren además de una investigación propia para desarrollar tratamientos específicos, accesibles y económicos que puedan ser adoptados por cada tipo de industria. El tratamiento primario consiste en la remoción, por medios físicos o químicos, de una parte sustancial del material sedimentable o flotante, reduciendo una fracción importante de la carga orgánica, que representa porcentajes significativos de los sólidos contaminantes. Entre los tipos de tratamiento primario se utilizan: la coagulación y la floculación. El objetivo principal del trabajo fue evaluar la eficacia de dos coagulantes en la reducción del potencial contaminante de los efluentes de la planta procesadora de frutas. Para realizar el tratamiento primario se ensayaron dos coagulantes químicos, el sulfato de aluminio como inorgánico y el Polycat CS-546o como orgánico. La eficacia se determinó con los ensayos de turbidez, demanda bioquímica de oxígeno y sólidos totales. Se concluye que el mejor coagulante para la remoción de la materia orgánica de los efluentes de la procesadoras de frutas es el Polycat CS-5460 habiendo logrado una remoción de turbidez de $98,1 \%$ y la reducción de la demanda bioquímica de oxígeno de $96,58 \%$, valores que se encuentran dentro de los límites permisibles para la descarga de efluentes industriales, según la legislación peruana.

Palabras clave: Tratamiento primario, floculación, coagulación, efluente industrial.

\section{SUMMARY}

Industrial effluents without treatment or supervision by the authorities pollute the environment. Solving the problem does not go only by the control, supervision and penalties for companies that generate effluents, require its own investigation as well as develop specific treatments, accessible and affordable that can be adopted by each industry. The primary treatment is removal by physical or chemical means a substantial part of sedimentable or floating material, reducing an important fraction of the organic load, which represents a significant percentage of solid contaminants. Within the types of primary treatment quote coagulation and flocculation. The main objective was to evaluate the efficacy of two coagulants in reducing the pollution potential of effluents from the fruit processing plant. To make the primary treatment: were assayed two chemical substances, aluminum sulfate as inorganic coagulant and other organic, the Polycat CS-5460. The effectiveness was determined by turbidity assays, biochemical oxygen demand and total solids. We conclude that the best coagulant for the removal organic matter in effluents from the processing of fruit is the Polycat CS-546o having achieved an important removal of turbidity (98.1\%) and reduction of biochemical oxygen demand (96.58\%) within allowable limits for discharge of industrial effluents, according peruvian law.

Keywords: Primary treatment, flocculation, coagulation, industrial effluent.

\section{INTRODUCCIÓN}<smiles>[CH]</smiles>
n países como el Perú, donde el desarrollo industrial es vertiginoso, se nota muy claramente un crecimiento en los niveles de contaminación ambiental, dañando los ecosistemas aledaños por la descarga de los efluentes industriales sin tratamiento previo ni control de las autoridades correspondientes. A pesar de la legislación existente para el control, no se ejecutan en todas las industrias por razones económicas, técnicas y administrativas, entre otras. El problema es agravado por el incremento de pequeñas y medianas industrias clandestinas, que al no estar registradas evaden dicho control, contribuyendo a la contaminación del medio ambiente. 
Resolver el problema planteado no pasa solamente por el control, supervisión y sanción a las empresas generadoras de efluentes (principalmente agua y materia orgánica), requiere además, debido a su gran diversidad, de una investigación propia para desarrollar tratamientos específicos, accesibles y económicos que puedan ser adoptados por cada tipo de industria. Estas investigaciones son denominadas "Estudios de tratabilidad de aguas residuales", diseñados con el objetivo de disminuir la capacidad contaminante por medio de uno o varios procesos de tratamiento; la finalidad es acondicionar el efluente a valores permisibles de carga orgánica y otros elementos contaminantes, para su vertido seguro a los cursos receptores de agua. Esto que parece tan lógico, raramente es realizado y es causa de innumerables fracasos en los sistemas de tratamiento ${ }^{(1)}$.

El tratamiento primario consiste en la remoción por medios físicos o mecánicos de una parte sustancial del material sedimentable o flotante, reduciendo una fracción importante de la carga orgánica, que representa porcentajes significativos de los sólidos contaminantes. Entre los tipos de tratamiento primario se utilizan: coagulación, floculación, flotación, sedimentación primaria, precipitación química, oxidación química y filtración ${ }^{(1,2)}$.

La floculación y coagulación son dos procesos dentro de la etapa de clarificación del agua. Ambos procesos se pueden resumir como una etapa en la cual las partículas se aglutinan en pequeñas masas (flóculos), hasta que sus pesos específicos superen al del agua y precipiten. La floculación tiene relación con los fenómenos de transporte dentro del líquido para que las partículas hagan contacto. Esto implica la formación de puentes químicos entre partículas, de modo que se forme una malla de coágulos de estructura tridimensional y porosa, hasta lograr formar un flóculo suficientemente grande y pesado como para sedimentar. La coagulación es la desestabilización de las partículas suspendidas, reduciendo las fuerzas de separación entre ellas. Se refiere a las reacciones que suceden al agregar un reactivo químico (coagulante) en el agua, originando productos insolubles. La coagulación comienza al agregar el coagulante al agua y dura fracciones de segundo ${ }^{(3-7)}$.
En ese contexto, el presente trabajo de investigación se hizo considerando los problemas de contaminación de nuestros ecosistemas por los efluentes agroindustriales no tratados. Se realizó el tratamiento primario de los efluentes de una planta procesadora de pulpa de frutas. El objetivo principal del trabajo fue evaluar la eficacia de dos coagulantes en la reducción del potencial contaminante de los efluentes de la planta procesadora de frutas - a través de la remoción de la materia orgánica, disminución de las unidades nefelométricas de turbidez (NTU) y de la demanda bioquímica de oxígeno (DBO) -, recuperando los grandes volúmenes de agua que pierde la planta en la limpieza y evacuación de sus efluentes con fines de reciclaje.

\section{MATERIALES Y MÉTODOS}

El presente trabajo de investigación fue realizado en el Laboratorio de Biotecnología y Microbiología Ambiental y en el Laboratorio de Bioprocesos Industriales de la Facultad de Ciencias Biológicas - Universidad Nacional Mayor de San Marcos.

Las muestras constituyeron los efluentes finales de la procesadora, las cuales fueron tomadas en recipientes estériles de $250 \mathrm{~mL}$ de capacidad, a diferentes tiempos durante el proceso. El homogeneizado de las muestras fue sometido a caracterización fisicoquímica considerando primordialmente los sólidos totales, turbidez, $\mathrm{pH}$ y demanda bioquímica de oxígeno.

\section{Caracterización fisicoquímica de los efluentes}

Para la determinación de las características de cada uno de los efluentes, éstos fueron sometidos a los siguientes ensayos:

\section{Determinación de pH}

Luego de homogeneizar la muestra se tomaron 100 $\mathrm{mL}$ y con ayuda del potenciómetro se determinó el valor de $\mathrm{pH}$.

\section{Determinación de azúcares totales (método Fehling Causse Bonnans) ${ }^{(8)}$.}

1. Se midieron $50 \mathrm{~mL}$ del efluente con una pipeta y se colocaron en un matraz aforado de $100 \mathrm{~mL}$.

2. Se añadieron $5 \mathrm{~mL}$ de $\mathrm{HCl} \mathrm{1:} \mathrm{1,} \mathrm{se} \mathrm{calentó} \mathrm{a}$ $70^{\circ} \mathrm{C}$ durante $15 \mathrm{~min}$. y se enfrió a temperatura ambiente.

3. Se neutralizó con $\mathrm{NaOH}$ usando fenolftaleína como indicador. 
4. Se completó el volumen a $100 \mathrm{~mL}$ con agua y se filtró.

5. Se colocó en una bureta la solución problema $y$, en una fiola de $250 \mathrm{~mL}, 5 \mathrm{~mL}$ de la solución de Fehling A y $5 \mathrm{~mL}$ de solución de Fehling B, se agregaron $20 \mathrm{~mL}$ de agua destilada y unas perlas de vidrio; se llevó a ebullición y vertió rápidamente la solución problema, con la ayuda de la bureta. A medida que la coloración cúprica (azul) se debilita, indica que la titulación está llegando a su fin. Se continuó la titulación hasta que el líquido que se encuentra sobre el precipitado rojo se tornó incoloro.

6. Se anotó el volumen de la muestra problema consumido en la titulación.

Cálculo:

\section{$\%$ A.R. = (f $/ \mathrm{mL}$ gastados $)_{100}$}

\section{Determinación de sólidos totales (método APHA) $)^{(8)}$}

Se agitó la muestra para eliminar los gases, luego se midieron $50 \mathrm{~mL}$ con pipeta volumétrica colocándolos en una cápsula de porcelana. Se evaporó la muestra en baño maría y se secó en estufa a $110{ }^{\circ} \mathrm{C}$ hasta peso constante. Al término, se pesó la cápsula con los sólidos.

\section{Determinación de la DBO (método de la A.O.A.C.) ${ }^{(4,5)}$}

Para esta prueba las muestras fueron procesadas inmediatamente llegadas al laboratorio $o$ en algunos casos luego de un máximo de 24 horas de almacenamiento en refrigeración.

Las muestras fueron colectadas en frascos de DBO de $300 \mathrm{mLy}$ transportadas al laboratorio en contenedores refrigerados.

La Demanda Bioquímica de Oxígeno fue determinada por el método propuesto en los Métodos estándares para el análisis de aguas y aguas residuales de la A.O.A.C. (1971). Las muestras diluidas al 5 y $25 \%$ y sin diluir, fueron incubadas a $20{ }^{\circ} \mathrm{C}$ en oscuridad durante 5 días, se determinó el contenido de oxígeno de cada uno de los frascos, antes y después de la incubación correspondiente, utilizando un electrodo de oxígeno después de un período de estabilización de 15 minutos. Los valores de la DBO fueron determinados considerando los valores iniciales y finales, aplicando la relación:

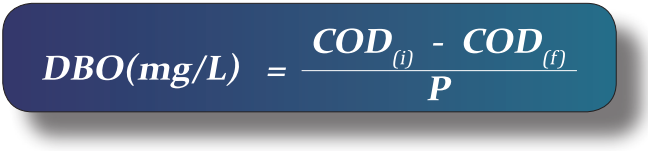

Donde:

$\mathrm{COD}_{(\mathrm{i})}=$ Concentración inicial de oxígeno disuelto.

$\mathrm{COD}_{(\mathrm{f})}=$ Concentración de oxígeno disuelto de las muestras luego de la incubación.

$\mathrm{P} \quad=$ Fracción decimal de muestra utilizada.

\section{Determinación de las NTU (Method 180.1 USEPA) ${ }^{(6)}$}

Una vez encendido el nefelómetro, se esperó hasta que se estabilice el equipo, luego se insertó la celda con el patrón correspondiente al rango de turbidez esperado para la muestra. Se ajustó la lectura al valor exacto del patrón con el control de calibración del equipo. Se colocó la muestra a medir en una celda nefelométrica, secando cuidadosamente la superficie externa y eliminando las burbujas que pudieran haber. Se retiró del nefelómetro la celda del patrón y se colocó la celda con la muestra. La lectura de turbidez se hizo directamente, expresada en NTU.

\section{Determinación de la eficacia de los coagulantes ${ }^{(2-6)}$}

Para realizar el tratamiento primario se ensayaron dos coagulantes químicos por el método De Jarras, recomendado por APHA, AWWA \& WPCF (1992), el sulfato de Aluminio $\left[\mathrm{Al}_{2}\left(\mathrm{SO}_{4}\right)_{3}\right]$ como inorgánico y el Polycat CS-546o como orgánico. La eficacia se determinó con los ensayos de turbidez (NTU), demanda bioquímica de oxígeno (DBO) y sólidos totales (SST).

\section{Coagulación - Floculación ${ }^{(1,4)}$}

El proceso de coagulación-floculación de aguas se realizó a nivel laboratorio. Primeramente se acondicionó el agua residual a $\mathrm{pH}$ 6,5 con la adición de ácido sulfúrico $1 \mathrm{~N}$ y a $\mathrm{pH}$ 9,o con la adición de $\mathrm{NaOH}$ $1 \mathrm{~N}$. Este líquido fue puesto bajo agitación a $100 \mathrm{rpm}$ y se le adicionaron simultáneamente los volúmenes adecuados de los coagulantes ya mencionados para obtener concentraciones de $50 \mathrm{mg} / \mathrm{L}$. Inmediatamente después se adicionó o,1 $\mathrm{mg} / \mathrm{L}$ del floculante anfotérico ${ }_{2} \mathrm{PNOD}_{2} \mathrm{R}$. La mezcla se agitó durante 30 segundos a $100 \mathrm{rpm}$ y después por 30 minutos a $30 \mathrm{rpm}$. Al 


\section{RESULTADOS}

Tabla 1. Caracterización de los efluentes de la procesadora de frutas antes del tratamiento

\begin{tabular}{|c|c|c|c|c|c|}
\hline № & Sustrato & NTU & $\begin{array}{c}\text { Sólidos totales } \\
(\mathrm{mg} / \mathrm{L})\end{array}$ & $\underset{\text { (mg/L) }}{\text { DBO }}$ & pH \\
\hline 1 & Mango & 168 & 2700 & 4380 & 5,5 \\
\hline 2 & Mango & 176 & 2760 & 4670 & 4,8 \\
\hline 3 & Mango & 183 & 3050 & 5020 & 4,3 \\
\hline 4 & Mango & 205 & 3800 & 5650 & 5,0 \\
\hline 5 & Mango & 179 & 2830 & 4880 & 4,6 \\
\hline 6 & Mango & 188 & 3530 & 5320 & 5,0 \\
\hline 7 & Mango & 185 & 3100 & 5100 & 5,1 \\
\hline 8 & Mango & 181 & 2990 & 4970 & 4,9 \\
\hline 9 & Mango & 192 & 3460 & 5400 & 4,7 \\
\hline 10 & Mango & 199 & 3480 & 5450 & 4,2 \\
\hline
\end{tabular}

Tabla 2. Caracterización de los efluentes de la procesadora de frutas después del tratamiento primario: coagulación-floculación

\begin{tabular}{|c|c|c|c|c|c|c|}
\hline \multirow{2}{*}{ № } & \multicolumn{3}{|c|}{$\mathrm{Al}_{2}\left(\mathrm{SO}_{4}\right)_{3}$} & \multicolumn{3}{|c|}{ Polycat CS-5460 } \\
\hline & NTU & ST & DBO & NTU & ST & DBO \\
\hline 1 & 5,2 & 162 & 218 & 4,0 & 145 & 158 \\
\hline 2 & 6,0 & 191 & 265 & 4,5 & 150 & 126 \\
\hline 3 & 6,5 & 180 & 300 & 3,0 & 135 & 192 \\
\hline 4 & 6,8 & 260 & 383 & 2,5 & 86 & 189 \\
\hline 5 & 5,5 & 223 & 338 & 3,0 & 155 & 176 \\
\hline 6 & 6,7 & 236 & 310 & 3,5 & 140 & 168 \\
\hline 7 & 6,1 & 242 & 347 & 3,1 & 148 & 159 \\
\hline 8 & 5,4 & 176 & 286 & 5,0 & 196 & 235 \\
\hline 9 & 6,0 & 219 & 305 & 2,8 & 169 & 171 \\
\hline 10 & 5,5 & 258 & 360 & 3,2 & 92 & 164 \\
\hline
\end{tabular}

Tabla 3. Porcentaje de remoción de materia orgánica usando Polycat-5460

\begin{tabular}{lccc}
\hline & ST & DBO & NTU \\
\hline Inicial & 3170 & 5084 & 185,6 \\
Final & 141,6 & 173,8 & 3,46 \\
Porcentaje & 95,5 & 96,58 & 98,1 \\
\hline
\end{tabular}

Tabla 4. Porcentaje de remoción de materia orgánica usando sulfato de aluminio $\mathrm{Al}_{2}\left(\mathrm{SO}_{4}\right)_{3}$

\begin{tabular}{lccc}
\hline & ST & DBO & NTU \\
\hline Inicial & 3170 & 5084 & 185,6 \\
Final & 214,7 & 311,2 & 5,97 \\
Porcentaje & 93,2 & 93,87 & 96,78 \\
\hline
\end{tabular}




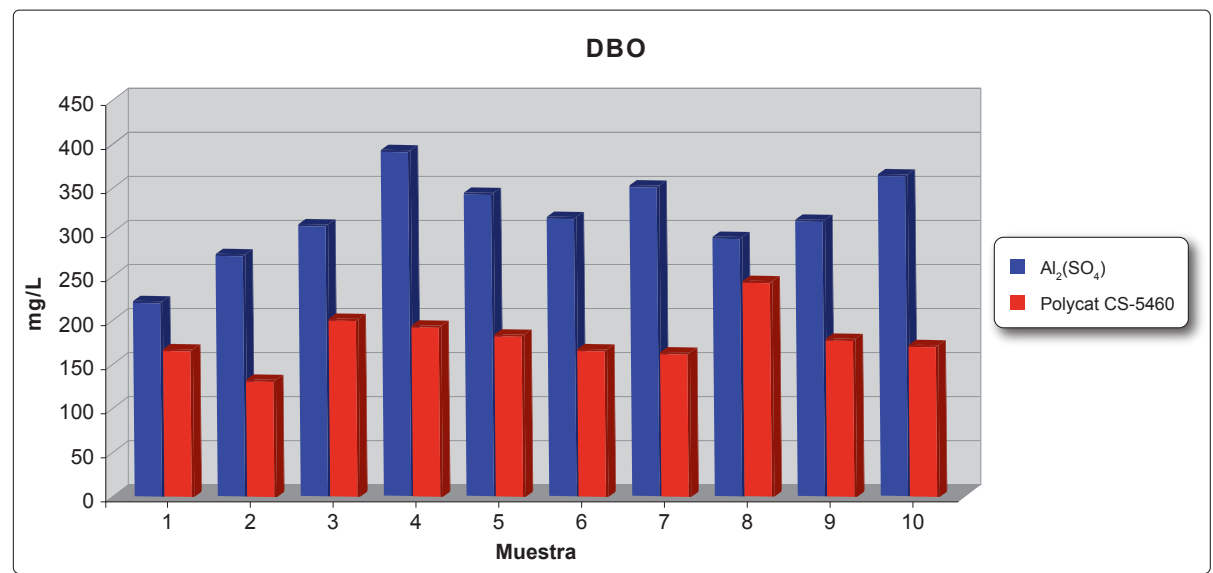

Figura 1. Reducción comparativa de la demanda bioquímica de oxígeno: Sulfato de aluminio $\mathrm{Al}_{2}\left(\mathrm{SO}_{4}\right)_{3}$ vs. Polycat CS-5460

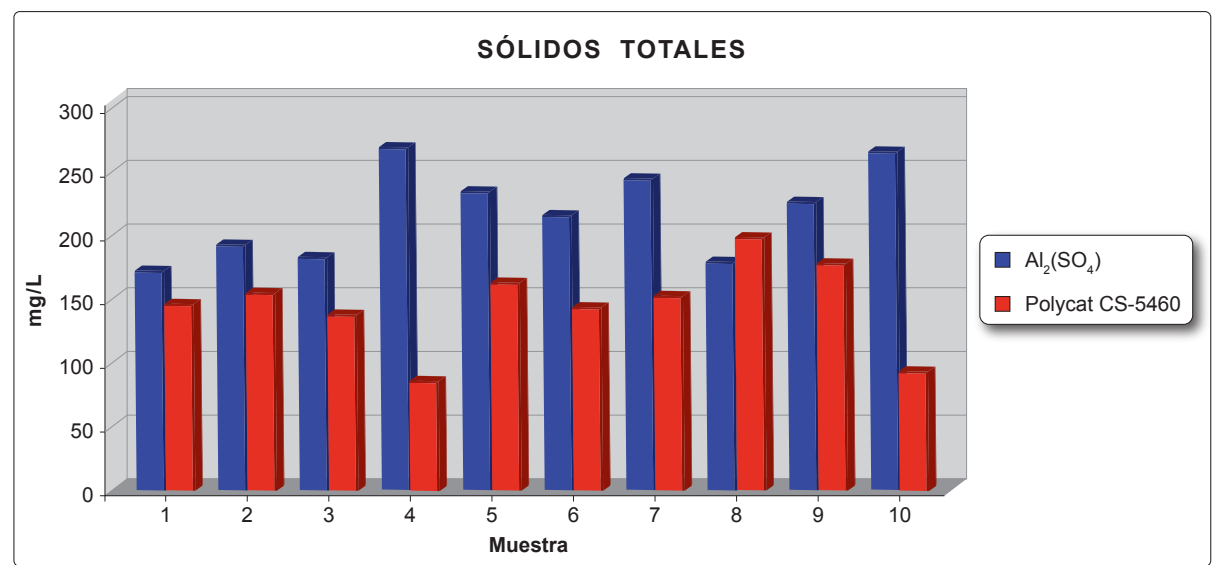

Figura 2. Reducción comparativa de los sólidos totales: Sulfato de aluminio $\mathrm{Al}_{2}\left(\mathrm{SO}_{4}\right)_{3}$ vs. Polycat CS-5460

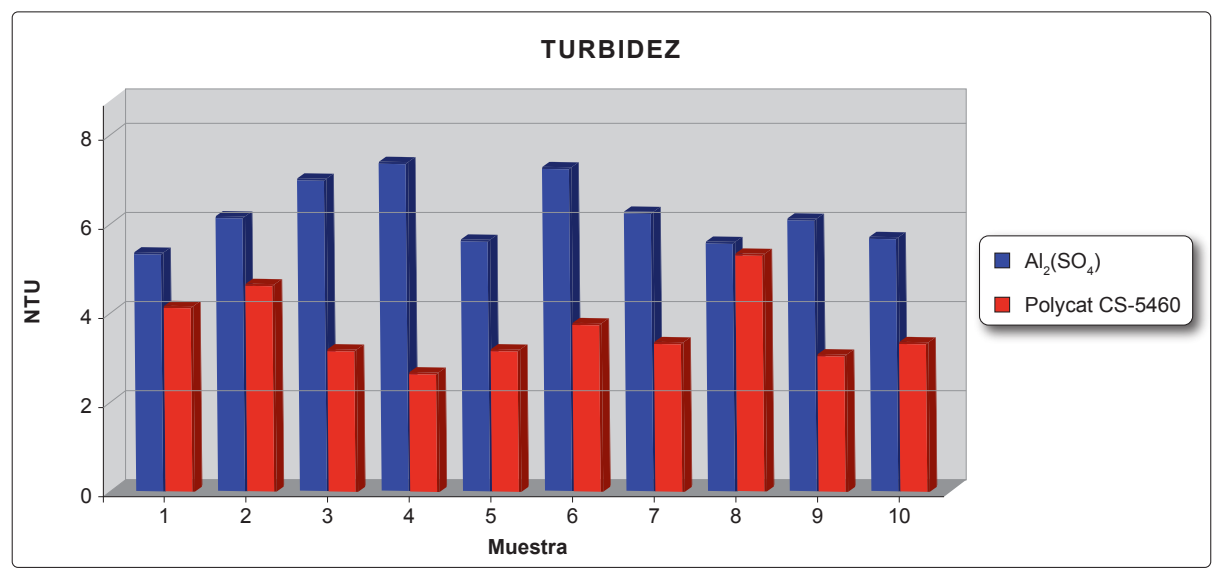

Figura 3. Reducción comparativa de turbidez: Sulfato de aluminio vs Polycat CS-5460 
término de este tiempo, se suspendió la agitación y se dejó sedimentar durante 30 minutos. El sobrenadante fue separado por decantación en peras verticales de decantación y analizados para determinar la DBO, SST y turbidez de los mismos.

\section{DISCUSIÓN Y CONCLUSIONES}

La caracterización de los efluentes de las plantas procesadoras de frutas varían en cada uno de los valores determinados, diferencias explicadas por el tipo de sustrato utilizado. En la planta, durante la investigación, se procesó mango. Los valores promedio encontrados con los cuales se trabajó se expresan en la tabla 1, siendo los más relevantes para la presente investigación la DBO 5084 y la turbidez $(185,6)$. En comparación, estos valores son bastante similares a los reportados por otras procesadoras de frutas; las variaciones se evidencian por la variedad de frutas utilizadas, el volumen procesado y la tecnología empleada.

Para el proceso de coagulación-floculación y sedimentación se ensayaron dos coagulantes químicos, habiendo sido elegido el producto orgánico por su mejor remoción de turbidez $(98,1 \%)$ y reducción de la demanda bioquímica de oxígeno (96,58\%), tal como lo demuestra en la tabla 3.

Los porcentajes de remoción encontrados en la presente investigación son similares a los reportados en otras investigaciones referentes al tratamiento primario, tal como la realizada por Meza y col. ${ }^{(9)}$ en el Instituto de Ingeniería-UNAM en la cual se presentan resultados del tratamiento mediante floculación-coagulación de los efluentes que descarga un reactor anaerobio que trata vinazas de tequila, con el fin de determinar la dosis efectiva de floculantes para eliminar el color y materia orgánica. Las pruebas se realizaron a nivel laboratorio en un equipo de jarras, con ayuda de floculantes tales como sulfato de aluminio y cloruro férrico, entre otros, probados de forma individual y en mezclas, variando la dosis y el $\mathrm{pH}$ de la vinaza. Los resultados obtenidos muestran que los floculantes más adecuados son: sulfato de aluminio y cloruro férrico, porque alcanzaron una eficiencia de remoción del 8o\%; sin embargo, está claro que los efluentes empleados por Meza y col. son diferentes en su composición química a los del presente estudio.

Ramírez y col. ${ }^{(10)}$, evaluaron el proceso de coagulación-floculación como una etapa de pretratamiento de las aguas residuales producidas en la Ciudad de México. El interés en esta investigación se centró en evaluar la eficiencia obtenida en el tratamiento con nuevos productos de coagulación-floculación comparada con la obtenida con productos comerciales de probada eficacia; llegándose a la conclusión que, de acuerdo a modelos matemáticos de remoción de parámetros fisicoquímicos, los mejores coagulantes y floculantes fueron PAX XL6o y ${ }_{2} \mathrm{PNOD}_{2} \mathrm{R}$, a $\mathrm{pH}$ 6,0 a una concentración de 20 $\mathrm{mg} / \mathrm{L}$ y $0,1 \mathrm{mg} / \mathrm{L}$, respectivamente. Con estos compuestos se obtienen eficiencias para SST del $88 \%$, color del $92 \%$, turbidez de $98 \%$ y DQO de $82 \%{ }^{(11-13)}$.

Se concluye que el mejor coagulante para la remoción de materia orgánica de los efluentes de procesadoras de frutas es el Polycat CS-546o, habiendo logrado una remoción de turbidez del $98,1 \%$ y una reducción de la demanda bioquímica de oxígeno de $96,58 \%$, valores que se encuentran dentro de los límites permisibles para la descarga de efluentes industriales, según la legislación peruana ${ }^{(14)}$.

Los bajos niveles de NTU encontrados, luego del tratamiento, permiten recuperar este recurso para las primeras etapas de lavado de la materia prima, las máquinas y equipos de la planta, reduciendo significativamente los costos de lavado a favor del proceso.

\section{REFERENCIAS BIBLIOGRÁFICAS}

1. Crites R, Tchobanoglous G. Sistemas de manejo de aguas residuales. Editorial McGraw-Hill Interamericana. Bogotá, 2000.

2. Environmental Protection Agency (EPA). Manual de diseño de pantanos construidos y sistemas de plantas acuáticas para el tratamiento de aguas residuales. Center for Environmental Research Information. Ohio, 1988.

3. Metcalf \& Eddy. Ingeniería de aguas residuales. 3ra ed. McGraw Hill. México, DF, 1996.

4. Araundel J. Tratamiento de aguas negras y efluentes industriales. 2da ed. Editorial Acribia. Zaragoza, 2002.

5. Metcalf \& Eddy. Ingeniería de aguas residuales: Tratamiento, vertido y reutilización. Volumen I. Mc Graw Hill. Madrid, 1995.

6. Centro Panamericano de Ingeniería Sanitaria y Ciencias del Ambiente (CEPIS). Manual de disposición de aguas residuales: Origen, descarga, tratamiento y análisis de las aguas residuales. CEPIS 
/ OPS / OMS. Tomo I. Lima, 1991.

7. Pérez PJ, Valverdú AA. Depuración y reutilización de aguas residuales. Encuentro medio ambiental almeriense: En busca de soluciones. Almería, 2003.

8. APHA. Standard methods for the examination of water and wastewater. 18th edition. Washington, 1994.

9. Meza PA, Briones MR, Ilangovan K. Floculacióncoagulación como postratamiento del efluente de un reactor anaerobio que trata vinazas tequileras. Coordinación de Bioprocesos Ambientales, Instituto de Ingeniería, UNAM. México, DF, 2004.

10. Ramírez R. Proceso de coagulación-floculación para el tratamiento de aguas residuales: Desarrollo y utilización de nuevos compuestos para la reducción de lodos. XII Congreso Nacional de Ingeniería Sanitaria y Ciencias Ambientales. 2000 Mar 21-24; Morelia. Programa de Ingeniería Química y Química Ambiental, Instituto de Ingeniería, UNAM. Mexico DF, 2000.

11. Olguín E, Peña E, Hernández E. Tecnologías ambientales para el desarrollo sustentable. Instituto de Ecología. Elsevier Science. México, 1994. p. 11-20.

12. Umaña R. Tratamiento de aguas residuales de la industria de curtiembre mediante sistema de lodos activados escala piloto. [Tesis]. Universidad de la Frontera Temuco. Chile, 2004.

13. Rueda-Becerril M. Evaluación del uso de un proceso químico en el tratamiento de las aguas residuales de una industria alimentaria. Facultad de Química UAEmex. Toluca, 2006. [En línea] consultado en octubre de 2009. Disponible en: http://www.uaemex.mx/red_ ambientales/does/

14. Decreto Supremo $\mathrm{N}^{\circ}$ 021-2009-VIVIEND. Aprueban Valores Máximos Admisibles (VMA) de las descargas de aguas residuales no domésticas en el sistema de alcantarillado sanitario. Lima 2009.

Manuscrito recibido el: 20/10/2010

Aceptado para su publicación el: 13/12/2010

\section{Correspondencia:}

Nombre: Mg. Mario Alcarraz Curi

Dirección: Jr. Puno 1002, Lima o1 - Perú

e-mail: biomac_2o@hotmail.com 